\title{
Implementation of Educational Communication Server for Smart Clothing Implementation
}

\author{
Jooyoung Ko*, Jaechang Shim**, Hyenki Kim* \\ Corresponding Author : HyenKi Kim, hkkim@anu.ac.kr \\ "Dept. of Multimedia Engineering, Andong National University, \\ *Dept. of Computer Engineering, Andong National University, \\ 1375 Gyeongdong-ro, Andong-City, Gyeongsangbuk-Do, Republic of Korea \\ sonice@anu.ac.kr,jcshim@anu.ac.kr,hkkim@andong.ac.kr
}

\begin{abstract}
IoT (Internet of Things) is a system of interrelated devices or internetworking of physical devices, vehicles, building etc. It also refers to an autonomous task processing system where information are obtained from things like sensor and process the information over a network without requiring human-to-human or human-to-computer interaction. IoT stands on 3 basic component called hardware, software and network. This study proposed a method of composing a server to implement a smart clothing network which comes under IoT. Here we used freeware processing program to suggest a simple method to implement Wi-Fi communication between a smart phone and a server and a serial communication with sensors. As a result, configuration of server was simplified and enabled a seamless communication between a smart phone and a sensor.
\end{abstract}

Keywords: Smart Clothing, IoT, Server, Processing

\section{Introduction}

Today, IoT (Internet of Things) is playing a very important role in our day to day life or we can say that it is an important keyword in our today's life. Ubiquitous computing environments provides the information of current environment condition of required location which helps people to understand and manipulate the environment according to their requirement. IoT (Internet of Things) mainly refers to automation system where there is no human interaction, it provides information need by the people and also to a network either human-to-computer or sensor-to-computers or sensors-to-sensors. For example, let's see the daily schedule of a farmer in a rural area under IoT environment [1]. Initially a farmer checks the environment condition with his current schedule and then he starts work at his work place using smart farming tools as required. If he use large equipment such as tractors then surrounding hazards are automatically checked for safety measure. It also checks farmer's health through a sensor attached to his arm or to the cloth, this sensor reads body temperature and alarm if any required measure needs to take off. It also notifies the family member of the farmer if any major incident occurs in the field. IoT also helps a farmer in buying farming products using smart phones and sell their product with the best deals.

Like this, Internet of Things obtains information from the surrounding environment with sensors and processes the obtained information to display on the smart phone. To implement Internet of Things, there needs to be adequate hardware, software, and network technologies. Our study suggests a communication server deployment method for communication between sensors-server, and smart phone-server in Internet of Things. This study has the advantage where students can make servers easily and in simple manner while implementing things with Internet of Things. When a server program is written with the suggested method, seamless communication with smart phones as well as 
sensors is enabled and it determines to provide an inexpensive and friendly environment to the students for implementing Internet of Things.

\section{Related Study}

Recently, computer network technology is changing rapidly and leaving a greater influence to our livelihood. Especially in Internet of Things environment, different types of object are connected in a network through which it became possible for us to obtain most of our necessary information from smartphones. Also in the case of a farmer, they can acquire weather information or crop prices through the smart phone and they also get help from emergency services if any such emergency situation occurs during their work [2]. In the field of medical, there are many active research for IoT systems that are ongoing for aiding patient health management. In the field of sports, various products have been introduced that can record and analyze the users' activity levels, temperature, and heart rate [3]. Smart clothing is a part of Internet of Things which is a system that can be worn or attached to people and it can measure and analyze biological changes of the user or activity levels [4].

Components of smart clothing are not different from the basic configuration of IoT. Smart clothing can also be known as Intelligent Clothing. An example of an early stage, smart clothing was introduced at the end of the 1990s which includes e-Textile, developed through cooperation between Levi's and Phillips which enable listening to music by attaching electronic components on apparel [5]. The application field of smart clothing recently expanded to military clothing, safety clothing, health management clothing, the sports field and many more in such like fields [3]. Application fields of smart clothing are shown in Figure 1.

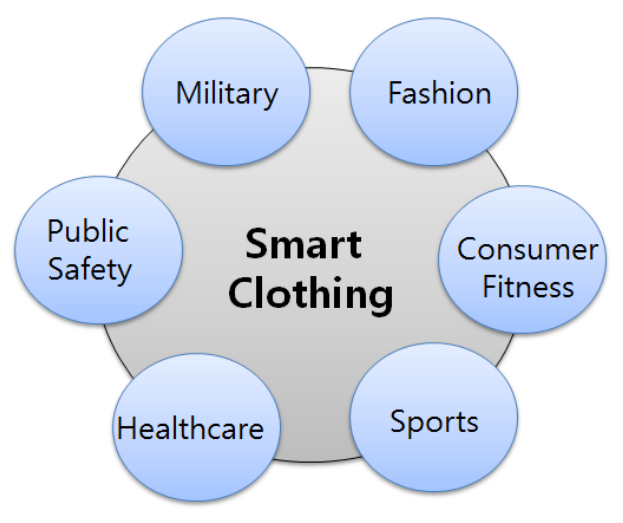

Figure 1. Components of Smart Clothing

To configure smart clothing, there needs to be a process of receiving data from sensors, processing of received data, and communicating to servers and smart phones to use the data in real world. Actually, there needs to be a process of delivering obtained data from sensors to servers either by wired or wireless communication network to implement smart clothing system.

This study suggests a method of writing a server program using a processing program as a method to implement wired or wireless communication for students to implement IoT and smart clothing systems. Processing programs can be used free of charge on various operating systems such as Linux, Macintosh and Windows. Serial communication through wired can be achieved but we propose for ZigBee as wireless communication hub so that communication process can be expanded through various devices. 


\section{Proposed Method}

\subsection{System Configuration}

The configuration of the system that composes smart clothing is shown in Figure 2. There needs to be IoT components hardware, software, and communication program.

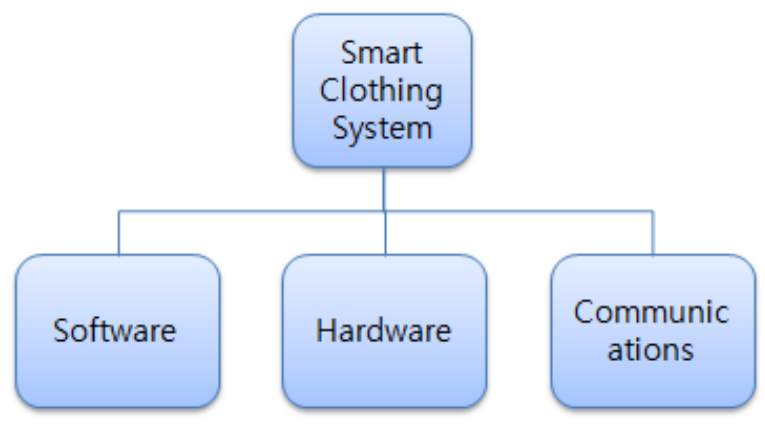

Figure 2. The Configuration of the System

\subsection{Program Utilized}

In this study Arduino is used as a hardware, which is widely used today in the field of education. Arduino is an open-source platform which is used for building electronics projects. It is famous for its simple programming and rich resources and can also be mount each other like bricks to add some more modules. It consists of microcontrollers and IDE which helps to upload computer code to physical board. It uses simplified version of $\mathrm{C}++$, making it easier to learn and use. The main characteristics of Arduino is, low price, low power and low speed. It is adequate for a system and such type of application with low data transfer speed. Arduino has 13 digital pins and 6 analog pins, it can use digital sensors as well as analog sensors. Analog sensors such as ambient light sensors and temperature sensors can be used.

Processing Program was used for the communication program [7]. Processing program can also be downloaded and used for free. Wi-Fi communication and serial communication setting is simple and server configuration is also simple. While the program is simple, it has excellent function and it is adequate for educational use.

The types used for hardware, software, and communication program in this study are shown in Figure 3.
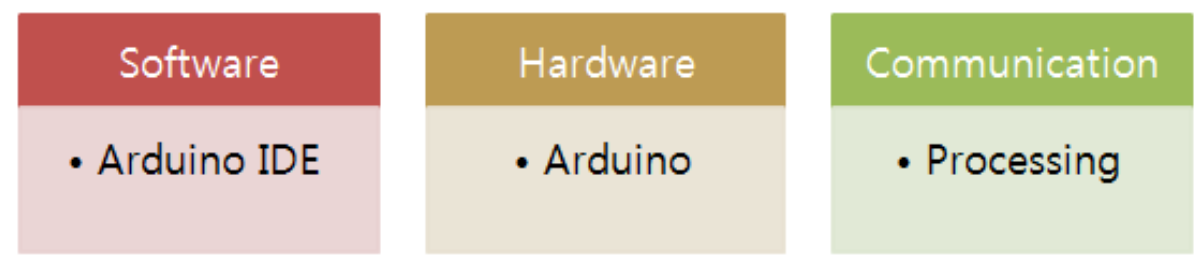

Figure 3. Programs for Communication

The role of software, hardware, and communication program is shown in Figure 4. Hardware configures the circuit and can control the input and output of digital and analog data. Software can write data processing and transfer programs and enables smart phone application development. The smart phone application development program used in the study was AppInventor [8]. AppInventor is an app development program developed by MIT Media Lab which can be easily used by nonprofessionals. 

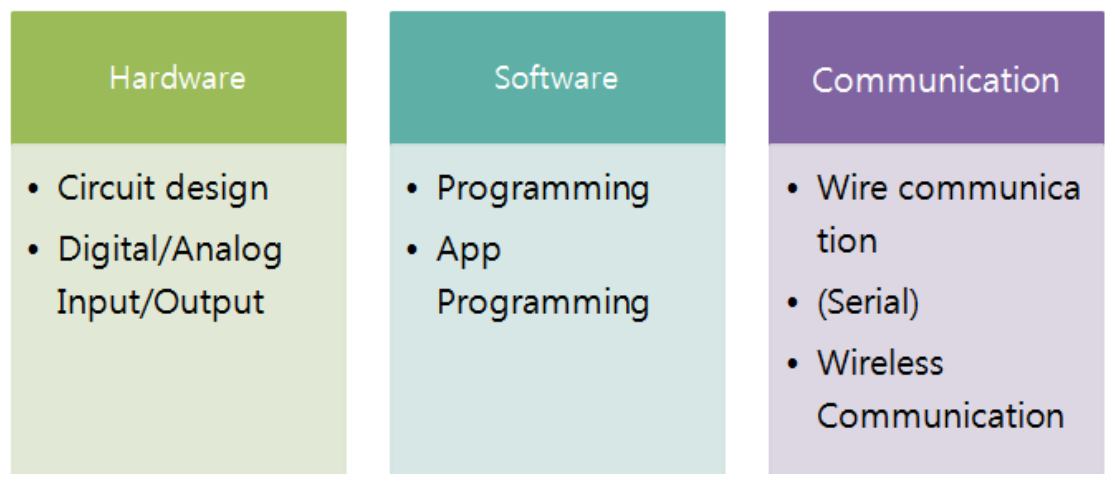

Figure 4. The Role of Software, Hardware, and Communication Program

\subsection{Educational Server Implementation}

Here, educational communication server is introduced to implement smart clothing. Figure 5 represents the communication direction between a smart phone and a server and between Arduino and server. Numbers indicated in Figure 5 represent communication direction with each device.

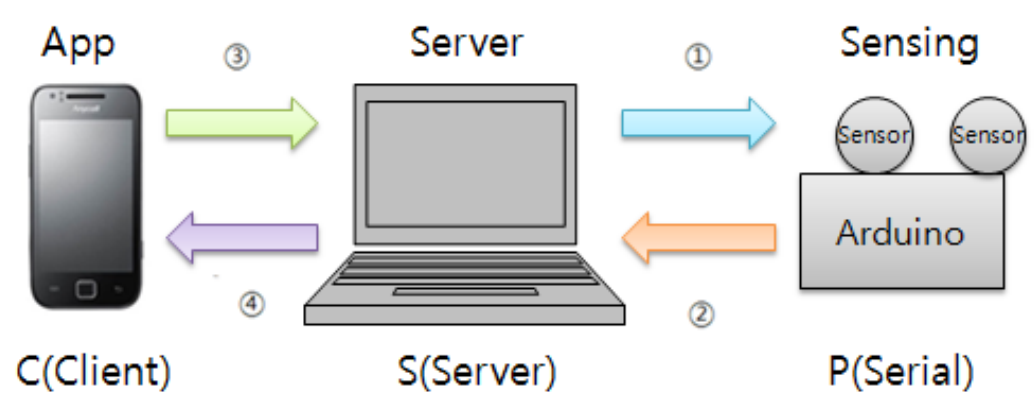

Figure 5. Communication Direction

Number (1) is the data transfer direction from a server to Arduino.

Number (2) is the data transfer direction from Arduino to a server.

Number (3) is the data transfer direction from a smart phone to a server.

Number (4) is the data transfer direction from a server to a smart phone.

Table 1 represents each communication method and type of communication.

Table 1. Communication Method and Type

\begin{tabular}{|l|c|c|c|c|}
\hline \multicolumn{1}{|c|}{ Num } & (1) & (2) & (3) & (4) \\
\hline $\begin{array}{l}\text { Communication } \\
\text { method }\end{array}$ & Wire & Wire & Wireless & Wireless \\
\hline $\begin{array}{l}\text { Communication } \\
\text { type }\end{array}$ & Serial & Serial & Wifi & Wifi \\
\hline
\end{tabular}

To implement a complete smart clothing system, data transferred from a smart phone must be delivered to hardware, and the sensed data must be delivered to the smart phone. 
To implement the system for education, it is important that the usage is simple. The study configured the server with processing language and enabled transmission of sensed data through serial communication to smart phones. When transmitting data from a server to Arduino, it is connected by serial cables and serial communication occurs.

\section{(1) Serial communication configuration}

The program that configures server with the processing program and transmits data through serial communication is as follows.

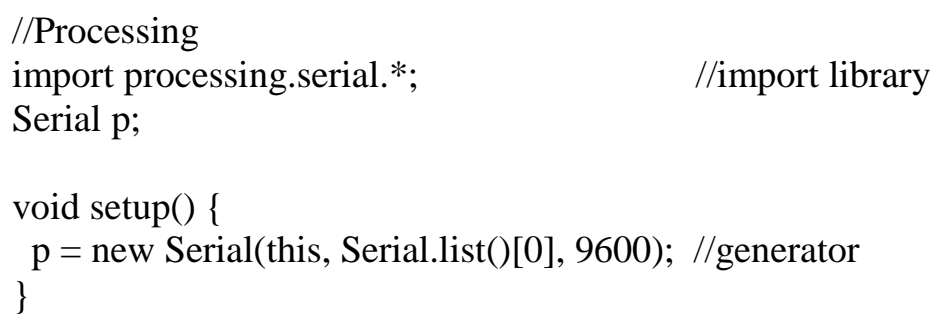

import processing.serial. ${ }^{*}$; First imports the internal library to use serial communication. Then, it declares Serial p; and declares generator in the void setup() function. When declaring the generator, serial port number was set and the data transfer speed was set as 9600 bps. Data transfer speed was identically configured in the Arduino program. The configuration part for Arduino program serial communication is as follows. Also in the Arduino program, it is configured in the setup() function.

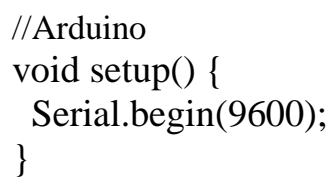

Communication speed can be changed according to the application program and it needs to be configured identically between the Arduino program and the processing program.

When transferring data from a server to Arduino, only serial communication is used and only simple commands have to be written. By writing in void draw() function, data can be transferred repeatedly.

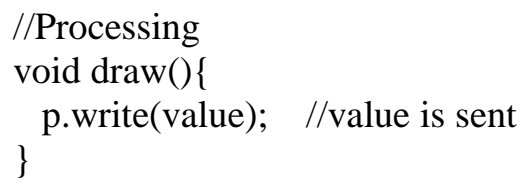

When controlling sensors by Arduino receiving data sent from servers, data can be received after confirming that serial communication is possible in the Arduino program. The program is as follows. Confirm that serial data is transferred in 'if (Serial.available() $>0)$ '. 'void loop()' function is a function that is repeatedly executed and it can continuously execute the command. 


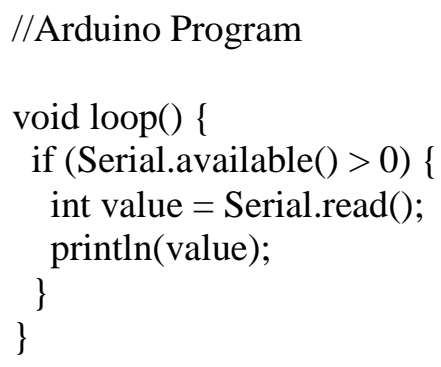

On the other hand, when sending data from Arduino to server, the server confirms there is serial data and stores data.

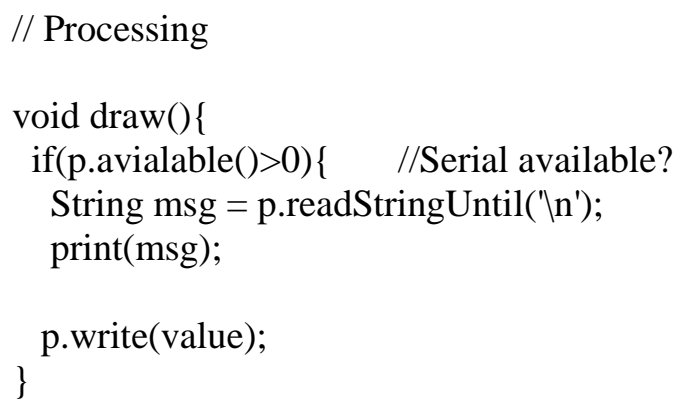

'p.avialable() $>0$ ' checks if there is data transferred through serial communication.

'String msg = p.readStringUntil( $\left(\mathrm{n}^{\prime}\right)$ )' ' is the process to leave only the data necessary among data transferred to serial.

For data transferred from Arduino program to serial, 'Serial. println(value);' was used. If number data was transferred using 'Serial. println(value);', it can be programmed with 'String msg = p.readString;'. In this study, while integer data was transmitted from Arduino, data was stored in string type including New Line ('In') using 'Serial.println()'.

(2) Wifi communication configuration

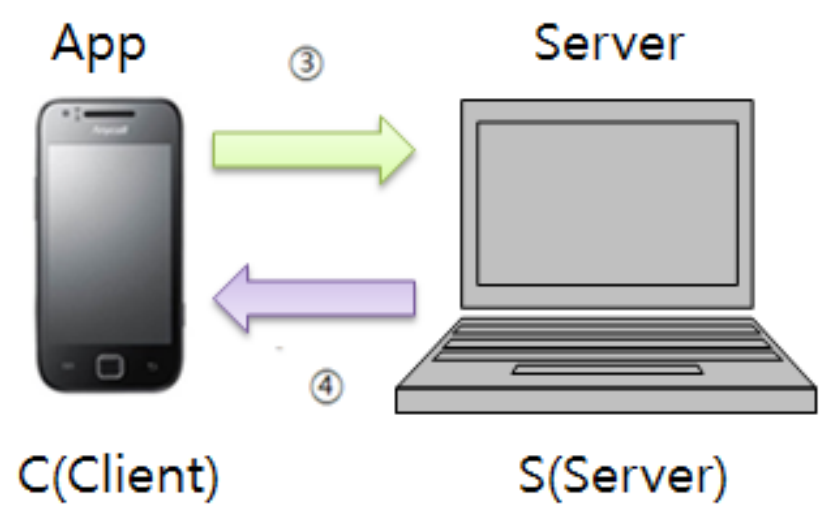

Figure 6. Wifi Communication 
As seen in Figure 6, a smart phone and a server attempted Wi-Fi communication. When IP address of each computer is designated and ports are selected, communication between smart phone and server is possible.

In this configuration, the smart phone becomes the client and the computer becomes server.

The processing internal library is imported like what follows below to configure client and server on the server program. Also we can be imported from the processing menu to Sketch> Internal library> Network.

import processing.net.*;

Server s;

Client c;

Then, server is generated within settings.

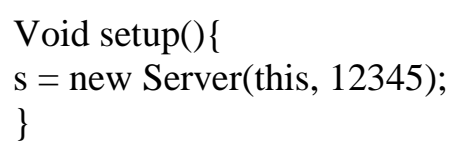

12345 is a random number representing a port where data can be sent from IP address.

The number 12345 must be the same transfer port when transferring data from smart phone app.

$s=$ new Server(this, 12345);

When data is transferred to the server, the server stores the data with 'String msg = c.readSTring();'

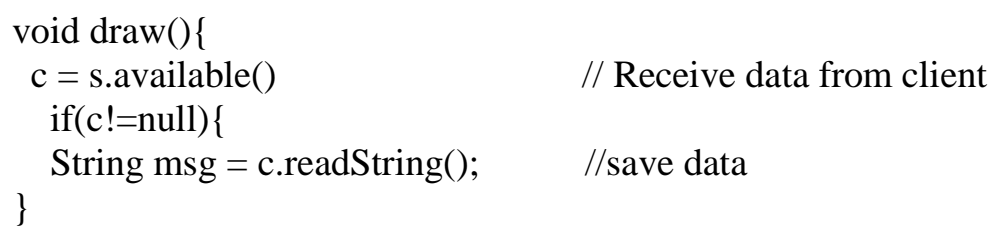

Here, the app on the smart phone is written using the App Inventor. To receive data in the server through the smart phone app, 'c.write(value);' is used on the server to transfer data.

To read data on the smart phone, smart phone displays standard HTML 5 rule is followed. HTML 5 is the most recent version of HTML (Hyper Text Markup Language) and it is a web standard applied on web pages as well as displays such as smart phones.

To display the data transferred from a server, the following standard code must be written. 


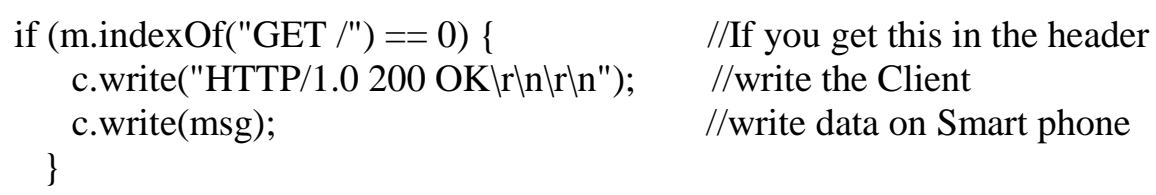

(3) ZigBee configuration

The study suggested a server program to implement smart clothing system. Communication between a server and microcontroller (Arduino) was wired communication method serial communication. In smart Clothing, the process of obtaining data from sensors through wireless network widens the actual application range. Therefore, the study researched wireless communication method between server and Arduino using ZigBee wireless communication module.

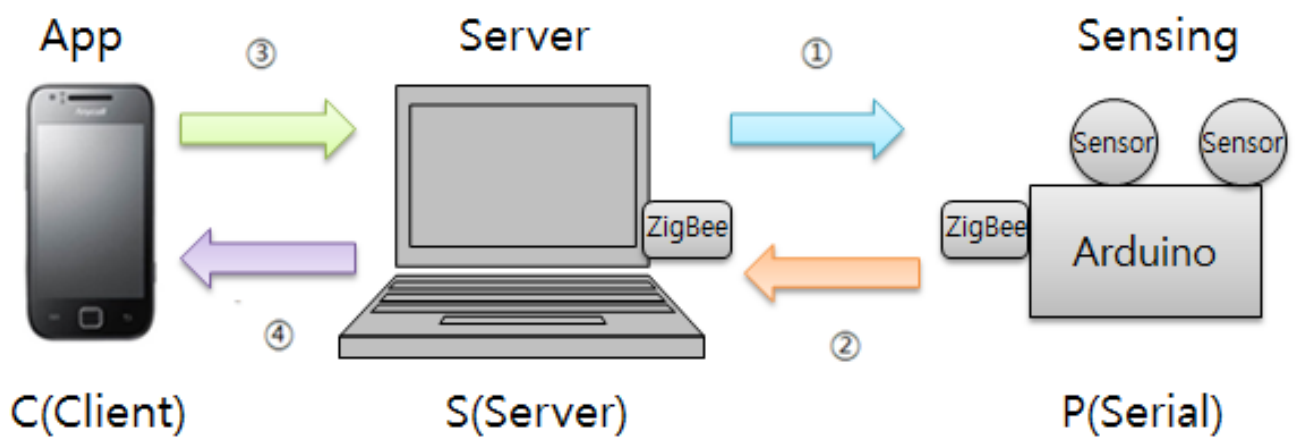

Figure 7. Communication Direction with ZigBee

In Figure 7, Number (1) transfers data from server to Arduino using ZigBee communication. Number (2) transfers data from Arduino using ZigBee communication.

Table 2. ZigBee Communication Methods and Forms

\begin{tabular}{|l|c|c|c|c|}
\hline \multicolumn{1}{|c|}{ Number } & $(1)$ & $(2)$ & $(3$ & (4) \\
\hline $\begin{array}{l}\text { Communication } \\
\text { method }\end{array}$ & Wireless & Wireless & Wireless & Wireless \\
\hline $\begin{array}{l}\text { Communication } \\
\text { type }\end{array}$ & ZigBee & ZigBee & Wifi & Wifi \\
\hline
\end{tabular}

ZigBee module can be used by connecting a ZigBee dongle on the server and connecting a ZigBee module on Arduino. If the channel of the ZigBee is the same, simple communication can be possible. 


\section{Experiment and Consideration}

Communication between a smart phone and a server through Wi-Fi and communication between server and Arduino was tested with our proposed system. AppInventor is used for the smart phone program. The app design and components made by using Up Inventor is shown in Figure 8. Necessary functions were included for data transferring when button is pressed, so two buttons and web is included as a required component. IP address is used while using web services.

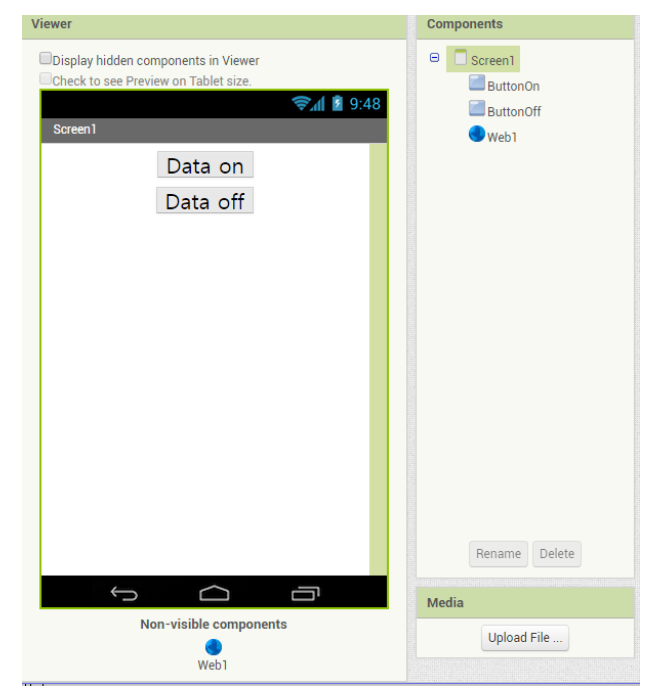

\section{Figure 8. Design and Components}

In App Inventor, commands are composed of blocks and when necessary blocks are arranged, the program is completed.

Figure 9 is an application program that transfers data written with blocks.
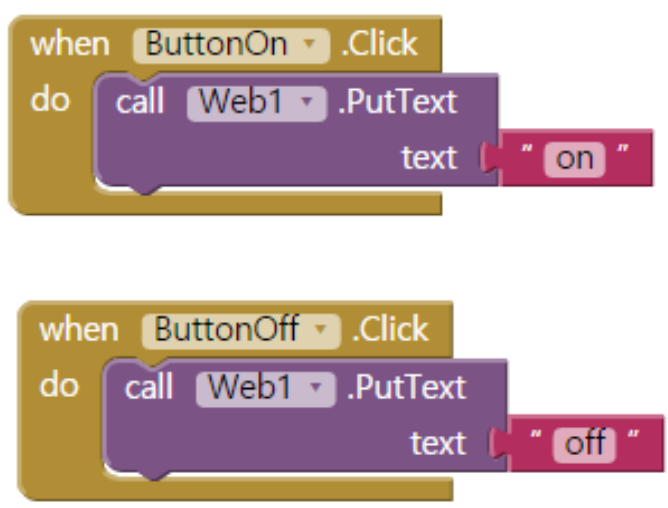

Figure 9. App Blocks

Figure 10 is the screen showing the application downloaded on the smart phone. The background color of the button and words can be changed when designing the application. 


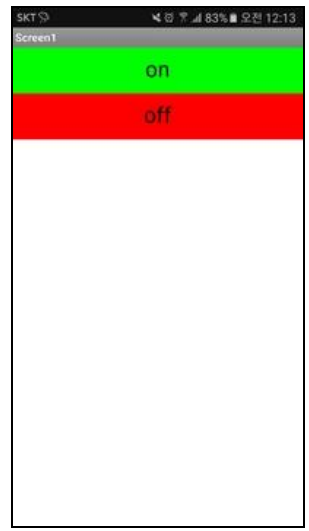

Figure 10. App Capture

After downloading and executing the program on the smart phone, after completion of the application, server program was completed on the computer by testing data transfer from a smart phone to a server. Data sent from the smart phone include Headers such as web addresses. To use only the data which we would use, parsing was conducted to extract only the necessary data.

The program parsing the data is as follows. As a result of various tests, for parsing method, the study confirmed the location of the point where there is data, the number of characters were counted, and according to the number, unnecessary characters cut off using substring(). Then,' p.write();' was used to transfer data to Arduino.

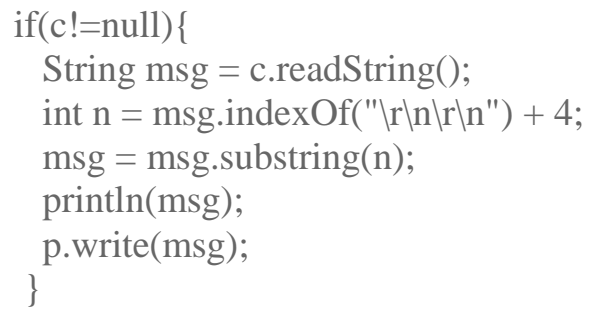

In the application program that enables receiving data from a smart phone to a server, Get block was used to enable receiving of data transferred to the web. Figure 11 utilizes Clock block to enable automatic data updating in pre-set time.
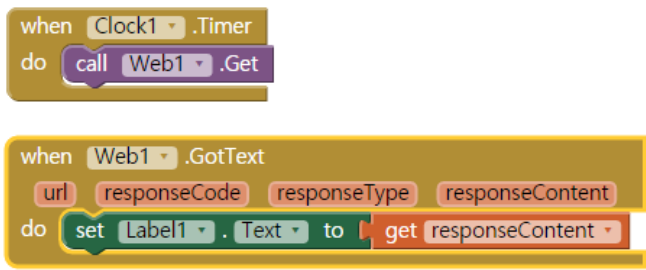

Figure 11. GotText Block 
The communication results between a smart phone to a server, server to Arduino, and smart phone Tarantino with the method suggested and implemented in the study is shown in Table 3.

Table 3. Communication Result

\begin{tabular}{|l|l|l|l|l|}
\hline & $\begin{array}{l}\text { Smart } \\
\text { Phone }\end{array}$ & Server & Arduino & ZigBee \\
\hline $\begin{array}{l}\text { Smart } \\
\text { Phone }\end{array}$ & & Succeed & Succeed & Succeed \\
\hline Server & Succeed & & Succeed & Succeed \\
\hline Arduino & Succeed & Succeed & & Succeed \\
\hline ZigBee & Succeed & Succeed & Succeed & \\
\hline
\end{tabular}

Data transfer was smooth in each communication direction and for communication between smart phone and Arduino, if the time setting was set to transfer with intervals over 0.5 seconds, the data was transferred without interruptions.

\section{Conclusion}

Smart clothing system is a part of IoT application and it is being researched and developed by various organizations. This topic attracts not only to the researcher but also to the students, which leads us to take this topic in the field of education.

During the training of the students, configuring and practicing to control sensors, transferring data to server and establishing a communication network to communicate with smart phones helps them to understand overall idea about IoT. On the other hand, students considered it difficult to configure servers and implement medication with smart phones. This study utilized free to use processing program with various functions to configure a server and to implemented Wi-Fi communication, serial communication, and ZigBee communication. The server written with processing program had the role of reliably transferring and receiving data. Therefore, it is determined that the server program suggested in this study is a method to simplify not only smart clothing system but also IoT system server implementation.

\section{References}

[1] J. Ko, J, Shim and K. Kim, "Smart Clothing System for Agricultural Workers" Conference Proceedings MITA 2016, Luang Prabang, Lao PDR, (2016), July 4-6.

[2] J. Ko , S, Yoon, K. Kim, “ Design and Implementation of Customized Farming Applications using Public Data” Journal of Korea Multimedia Society Vol. 18, No. 6 ( 2015), pp. 772-779.

[3] M. G. Honarvar, M. Latifi, "Overview of wearable electronics and smart textiles", The Journal of The Textile Institute, vol. 107, no.1 , (2016), pp. 1754, 2340.

[4] J. Ko, Smart Clothing: Human, Clothing, Computer, Journal of Multimedia, Vol.17, No.3_4, (2013), pp.41-50.

[5] L.v. Langenhove and C. Hertleer, "Smart clothing: a new Life", International Journal of Clothing Science and Technology, vol. 16, no. 1/2, (2004), pp. 63-72.

[6] J. Shim, J. Ko, Y. Lee, W. Jung, "2 ${ }^{\text {nd }}$ Edition Funny Arduino", Hantee Media, Seoul, Korea ,(2013)

[7] J Shim, J. C. Shim, Y. Yang, "Yagum Yagum Processing”, Chaos Book, Paju,Korea, (2013).

[8] J. Shim, J. B. Shim, J, Ko, J. C. Shim, Guljam App Inventor”, , Chaos Book, Paju, Korea, (2014). 


\section{Authors}

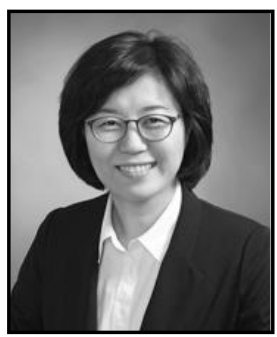

Jooyoung Ko, She received her Ph.D. in Multimedia Engineering from Andong National University in 2010. She currently works at Institute for Education Development in Andong National University. She is interested in Smart clothing for elderly people.

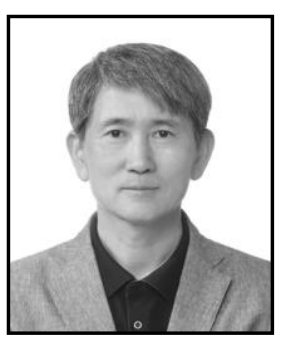

Jaechang Shim, Jaechang Shim is a Professor in the Department of Computer Engineering at Andong National University. His research interests include pattern recognition, image processing, embedded vision system, biometric authentication, and 3D object recognition. He received the Best Paper Award from IAPR in 1998. He is also a co-founder of PAMI Inc. since 1998. He received his B.S., M.S., and Ph. D. in electronics engineering from Kyungpook National University, Korea, 1987, 1990, and 1993 respectively

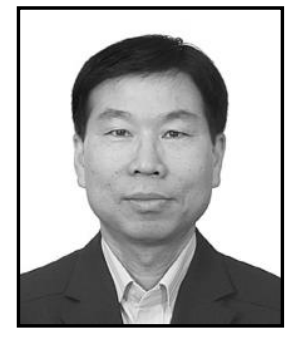

Hyenki Kim, He received his B.S. and M.S. degree in Electronics Engineering from Kyungpook National University, Korea in 1986 and 1988 respectively. He received his Ph.D. in Electronics Engineering from Kyungpook National University, Korea in 2000. He joined Andong National University in 2002, where he is currently a professor at Dept. of Multimedia Engineering in Korea. His research interests include Multimedia System, mobile app. and Multimedia Communication. 